\title{
Explorando a Gamificação como Abordagem Não Restritiva ao Gaming the System e Help Refusal em Sistemas Tutores Inteligentes
}

\author{
Otávio Bastos Azevedo ${ }^{1}$, Patrícia A. Jaques ${ }^{1}$ \\ ${ }^{1}$ Universidade do Vale do Rio dos Sinos (UNISINOS) \\ São Leopoldo - RS - Brasil \\ obazevedo@edu.unisinos.br, pjaques@unisinos.br
}

\begin{abstract}
Intelligent Tutoring Systems (ITSs) have shown to be almost as effective as one-to-one tutoring. Nonetheless, improper use of the ITS intelligent assistance can lead students to unwanted behaviors, such as Gaming the System or Help Refusal. This paper presents the use of gamification as an approach to prevent gaming the system and help refusal. This system was integrated into a step-based algebraic ITS and it was evaluated in an experiment, for 6 weeks, involving 60 students from the $7^{\text {th }}$ year of elementary school. The results of this research evidenced that gamification can be used to reduce the behavior of Gaming the System, but we did not observe significant reductions in the Help Refusal behavior.
\end{abstract}

Resumo. Os Sistemas Tutores Inteligentes (STIs) têm se mostrado quase tão efetivos quanto à instrução individual. Entretanto, a utilização indevida da assistência inteligente do STI pode levar os alunos a comportamentos indesejados como Gaming the System ou Help Refusal. Este trabalho apresenta uma proposta de solução para o problema, a partir do emprego de Gamificação para prevenir Gaming the System e Help Refusal. Este sistema foi integrado a um STI algébrico baseado em passos e utilizado em uma avaliação experimental envolvendo 60 alunos do $7^{\circ}$ ano do ensino fundamental. Os resultados dessa pesquisa evidenciam que a gamificação pode ser empregada para reduzir o comportamento de Gaming the System, porém não observamos reduções significativas no comportamento de Help Refusal.

\section{Introdução}

A Gamificação tem a sua origem nos jogos digitais e pode ser definida como a utilização de elementos de jogos em sistemas ou contextos que não são jogos, a fim de incentivar a participação de seus usuários [KAPP, 2012]. Esta técnica pode ser aplicada nas mais diversas áreas e vem obtendo sucesso especialmente em ambientes de aprendizagem [HUANG, 2013]. Os elementos de Gamificação podem ser tanto aplicados nas aulas convencionais (por exemplo: oferecer recompensas para os alunos após a resolução de exercícios), como também nos Sistemas Tutores Inteligentes [GONZÁLEZ et al., 2014].

Um Sistema Tutor Inteligente (STI) é um programa computacional destinado a auxiliar os alunos no processo de ensino-aprendizagem, utilizando técnicas de inteligência artificial e teorias pedagógicas para adaptar-se às características desses estudantes, proporcionando assim assistência individualizada [VANLEHN, 2006; 
VII Congresso Brasileiro de Informática na Educação (CBIE 2018)

Anais dos Workshops do VII Congresso Brasileiro de Informática na Educação (WCBIE 2018)

WOOLF, 2010]. Os STIs baseiam-se na estratégia de ensino dos professores particulares e têm se mostrado quase tão efetivos quanto à instrução particular [KULIK \& FLETCHER, 2016].

Os tutores inteligentes oferecem uma assistência individualizada aos seus alunos a partir do feedback mínimo, que informa se o passo resolvido ou a resposta final está correto ou incorreto. Caso a resposta do aluno esteja errada, o sistema fornece um feedback de erro que retorna uma dica para o aluno corrigir a sua resposta. Os estudantes também podem solicitar ajuda manualmente; nesse caso, o sistema retorna uma mensagem de ajuda para o aluno, orientando-o a resolver o próximo passo do exercício ou chegar na resposta final. Tanto os feedbacks quanto as dicas são fornecidos pelo sistema de ajuda do STI, o qual interage diretamente com o aluno para efetuar a correção de suas respostas e ajuda-o a prosseguir na resolução do exercício quando for necessário.

Apesar de sua eficiência, o rico sistema de ajuda e feedback dos Sistemas Tutores Inteligentes pode levar os alunos a comportamentos indesejados, como o Gaming the System (GTS) e o Help Refusal (HR). O Gaming the System ocorre quando o estudante abusa do sistema de ajuda oferecido (Help Abuse, em inglês), com o intuito de chegar à resposta final sem reflexão prévia [BAKER, 2006]. Ainda segundo Baker (2006), o comportamento de tentativa e erro (que envolve várias tentativas falhas de resolver o exercício sem uma pausa para analisar o motivo do erro) também está relacionado ao Gaming the System. Por outro lado, um aluno apresenta o comportamento de Help Refusal quando ele se recusa a pedir ajuda ao sistema, geralmente para não se sentir inferior aos outros colegas e por acreditar erroneamente que pedir ajuda demonstra falta de inteligência [VANLEHN, 2006].

Ambos os comportamentos anteriores prejudicam o aprendizado do conteúdo. Baker (2006) observou que os estudantes que praticaram Gaming the System obtiveram um desempenho inferior na avaliação em relação aos alunos que não apresentaram esse comportamento. Os alunos que não pedem ajuda quando estão com dificuldades também podem apresentar um menor desempenho, inclusive nas sessões de utilização do STI, uma vez que eles não conseguirão avançar de forma adequada nas atividades propostas.

Nesse contexto, o objetivo geral deste trabalho é analisar como os elementos de gamificação podem minimizar a resistência dos alunos de solicitar ajuda, de forma que não os direcione ao Gaming the System. Para alcançar este objetivo, um modelo computacional de gamificação foi desenvolvido e integrado a um STI baseado em passos, que assiste os estudantes a resolverem equações algébricas de primeiro grau.

\section{Trabalhos Relacionados}

Esta seção apresenta os trabalhos que possuem um STI e/ou que tratam o comportamento de Gaming The System a partir da Gamificação, com o objetivo de salientar o diferencial do trabalho proposto em relação ao estado da arte.

O E-Game [PEDRO, 2016] é um STI que ajuda os alunos a responderem questões de múltipla escolha de matemática. O seu objetivo foi utilizar a Gamificação para reduzir o comportamento de GTS. Os resultados de um experimento com duração de 17 meses mostraram um forte impacto da gamificação na diminuição do GTS, que foi observado pelo tempo de permanência do aluno na resolução de problemas matemáticos. Os alunos ficaram mais tempo resolvendo os exercícios ao usar o sistema gamificado (67\% a mais do que no tutor sem elementos de gamificação). 
VII Congresso Brasileiro de Informática na Educação (CBIE 2018)

Anais dos Workshops do VII Congresso Brasileiro de Informática na Educação (WCBIE 2018)

Uma versão gamificada do Lynnette [LONG \& ALEVEN, 2014], um STI baseado em passos que assiste os estudantes a resolverem equações de $1^{\circ}$ grau - foi avaliada para verificar se a gamificação leva os alunos a um maior engajamento e melhor aprendizado. Embora os alunos apreciaram os elementos de gamificação inseridos no sistema, a técnica utilizada não proporcionou maior engajamento ou ganhos de aprendizado.

O STI MathDungeon [FAGHIHI et al., 2014] atua no ensino de matemática e foi utilizado em conjunto com técnicas de gamificação e animações artísticas para criar lições de matemática em nível universitário que sejam divertidas, combinando jogo com aprendizado. $\mathrm{O}$ grupo dos alunos que utilizaram o MathDungeon em um experimento obteve um desempenho maior em relação aos grupos de controle (um dos grupos utilizou a ferramenta ALEKS que avalia o conhecimento do aluno e o instrui sobre os tópicos que está mais apto para aprender, e o outro grupo não utilizou nenhuma ferramenta de tutoria).

O MeuTutor [NETO et al., 2015; DE SANTANA et al., 2016], um STI que auxilia os alunos do ensino fundamental e médio no aprendizado de português e matemática, foi empregado em dois estudos relacionados à gamificação. $\mathrm{O}$ primeiro mostrou que a gamificação contribuiu para o aumento de desempenho dos estudantes do $1^{\circ}$ ano do ensino médio na aprendizagem de matemática. O segundo trabalho mostrou que os alunos que estão na fase inicial de uso do tutor interagiram com um maior número de elementos de gamificação, enquanto que os alunos mais avançados interagiram com os elementos que afetam o seu desempenho de forma mais significativa.

Todos os trabalhos anteriores utilizaram a gamificação em STIs para promover o engajamento e aprendizado dos alunos, sendo que o E-Game [PEDRO, 2016] foi o único trabalho que também tratou o comportamento de GTS. O principal diferencial do nosso trabalho é que ele trata simultaneamente os comportamentos de GTS e HR a partir da Gamificação em um STI step-based, de maneira que minimize a resistência dos alunos de solicitar ajuda sem direcioná-los ao GTS. O trabalho proposto também oferece uma certa quantidade de dicas e erros sem custos de pontuação, o que incentiva os estudantes a pedirem ajuda quando tiverem dificuldades. Além disso, os erros gratuitos estimulam os alunos a tentarem resolver sozinhos os exercícios quando eles se sentirem aptos, de modo que não fiquem com medo de errar para não perder pontos, uma vez que os erros também fazem parte do aprendizado.

\section{Trabalho Desenvolvido}

Neste trabalho foi usado o STI PAT2Math, que trabalha com o conteúdo de equações de $1^{\circ}$ grau. O PAT2Math fornece um feedback mínimo para cada um dos passos resolvidos e uma mensagem de ajuda (feedback de erro) em caso de erro [JAQUES et al., 2013]. Os alunos também podem pedir ajuda caso não saibam como prosseguir na resolução da equação. As equações estão distribuídas em planos de aula, que as classificam de acordo com o nível de dificuldade. Esses níveis estão classificados em ordem crescente, sendo o primeiro plano de aula o mais simples (com equações no formato $\mathrm{x}+\mathrm{b}=\mathrm{c}$, onde $\mathrm{b}$ e $\mathrm{c}$ são constantes) e o último é o mais complexo, envolvendo números fracionários e a propriedade distributiva. Os alunos devem resolver todas as equações de um plano para liberar o próximo, mas, dentro de um plano, possuem liberdade para escolher a ordem em que resolverão as equações.

Foram implementadas três versões do STI para o experimento deste projeto: totalmente gamificada, parcialmente gamificada e sem gamificação. Ambos os grupos 
VII Congresso Brasileiro de Informática na Educação (CBIE 2018)

Anais dos Workshops do VII Congresso Brasileiro de Informática na Educação (WCBIE 2018)

gamificados contaram com a distribuição dos planos de aula por níveis de dificuldade e com as pontuações por equação. Os alunos só podiam acessar o próximo plano de aula após resolver todas as equações do anterior (com exceção do primeiro, que já vem disponível desde o início). O desbloqueio dos níveis segue o mesmo sistema: um nível só é liberado após o estudante completar todos os planos do nível anterior. No grupo totalmente gamificado também estavam presentes o sistema de dicas e erros gratuitos (que não ocasionam perda de pontos), a pontuação total e por nível de dificuldade. $\mathrm{O}$ grupo sem gamificação contou apenas com as funcionalidades básicas do tutor: assistência para a resolução passo-a-passo das equações, equações distribuídas em planos de aula que poderiam ser acessados em qualquer ordem e com os sistemas de dicas e exemplos trabalhados.

O Quadro 1 apresenta um resumo das características, diferenças e semelhanças de cada versão, e a Figura 1 mostra a comparação de suas interfaces gráficas.

\section{Quadro 1. Comparação dos recursos}

\begin{tabular}{|c|c|c|c|}
\hline & \multicolumn{3}{|c|}{ Funções presentes nos três sistemas } \\
\hline & \multicolumn{3}{|c|}{$\begin{array}{l}\text { Novo sistema de exemplos trabalhados, onde o aluno decide no início de cada plano de aula se deseja } \\
\text { conferir o exercício resolvido (anteriormente a visualização do exemplo trabalhado era obrigatória) }\end{array}$} \\
\hline & Gamificação Completa & Gamificação Parcial & Sem Gamificação \\
\hline Dicas & $\begin{array}{l}\text { Conta com dicas gratuitas de acordo com o } \\
\text { nível de complexidade do plano de aula. As } \\
\text { dicas excedentes custam } 3 \text { pontos. }\end{array}$ & $\begin{array}{l}\text { Cada dica solicitada } \\
\text { custa } 3 \text { pontos, sem dicas } \\
\text { gratuitas. }\end{array}$ & \multirow{4}{*}{$\begin{array}{l}\text { Sem pontuação e outras } \\
\text { recompensas ou } \\
\text { mecanismos de jogo (o } \\
\text { post-it possui apenas o } \\
\text { botão de dica e a barra de } \\
\text { progresso), dessa forma as } \\
\text { dicas, erros e exemplos } \\
\text { trabalhados não terão } \\
\text { custos }\end{array}$} \\
\hline Feedbacks de Erro & $\begin{array}{l}\text { Conta com erros sem perda de pontos de } \\
\text { acordo com a dificuldade do plano de aula. } \\
\text { Os erros excedentes custam } 5 \text { pontos }\end{array}$ & $\begin{array}{l}\text { Cada erro custa } 5 \text { pontos, } \\
\text { sem erros gratuitos. }\end{array}$ & \\
\hline $\begin{array}{c}\text { Exemplos } \\
\text { Trabalhados }\end{array}$ & $\begin{array}{l}\text { Primeira visualização gratuita em cada plano } \\
\text { de aula, as demais custam } 8 \text { pontos }\end{array}$ & $\begin{array}{l}\text { Todas as visualizações } \\
\text { são gratuitas }\end{array}$ & \\
\hline Pontuação & Total, por nível de dificuldade e por equação & Somente por equação & \\
\hline $\begin{array}{l}\text { Níveis de } \\
\text { dificuldade }\end{array}$ & \multicolumn{2}{|c|}{$\begin{array}{l}\text { Cinco níveis de dificuldade: Básico, Intermediário, Avançado, Expert e } \\
\text { Season Finale. Cada nível, com exceção do último que contém equações } \\
\text { de todos os níveis anteriores, possui de quatro a cinco planos de aula. }\end{array}$} & $\begin{array}{l}\text { Sem definição de níveis de } \\
\text { dificuldade }\end{array}$ \\
\hline Planos de aula & \multicolumn{2}{|c|}{$\begin{array}{l}\text { Os alunos devem resolver todas as equações de um plano para liberar o } \\
\text { próximo. Os títulos dos planos de aula assemelham-se às fases de um jogo } \\
\text { eletrônico }\end{array}$} & $\begin{array}{l}\text { Os alunos podem resolver } \\
\text { os planos em qualquer } \\
\text { ordem. Os títulos indicam } \\
\text { apenas o seu conteúdo }\end{array}$ \\
\hline
\end{tabular}

\section{Avaliação}

A avaliação teve como objetivo verificar o impacto dos elementos de gamificação inseridos (dicas e erros gratuitos, distribuição dos planos de aula em níveis de dificuldade e exibição da pontuação total e por nível) na redução dos comportamentos de Gaming the System e Help Refusal, no aumento do engajamento dos alunos em utilizar o tutor para estudar matemática e na melhoria no desempenho escolar dos estudantes.

O experimento foi realizado em uma escola particular da cidade de São Leopoldo, RS e contou com a participação de três turmas do sétimo ano do ensino fundamental (60 alunos no total, com idade variando de 12 a 14 anos) durante seis semanas, onde cada semana teve a duração de um período de 50 minutos por turma. Na primeira semana foi aplicado o pré-teste, para verificar os conhecimentos prévios dos alunos antes da utilização do programa. Nas quatro semanas seguintes, os alunos utilizaram o sistema desenvolvido no laboratório de informática do colégio, que contava com um computador por aluno. Na última semana foram aplicados o pós-teste e o questionário). 
VII Congresso Brasileiro de Informática na Educação (CBIE 2018)

Anais dos Workshops do VII Congresso Brasileiro de Informática na Educação (WCBIE 2018)

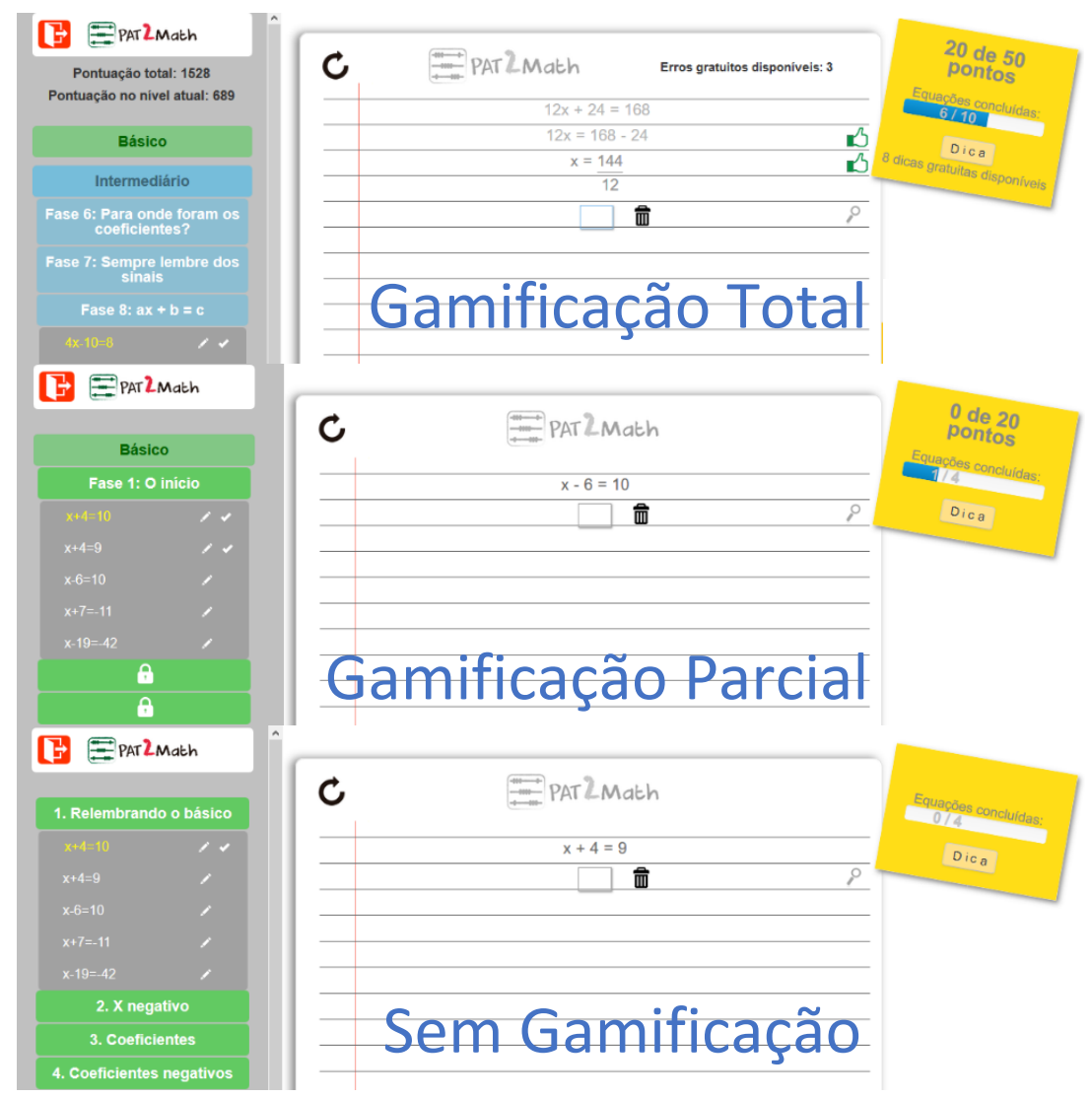

Figura 1. Comparação das interfaces gráficas

Devido às grandes diferenças de interface gráfica entre as versões gamificadas e as que não foram gamificadas, os alunos não foram distribuídos nos grupos de forma totalmente aleatória, uma vez que se houvesse a percepção de que existem versões diferentes do programa, a avaliação seria impactada negativamente (por exemplo, o efeito Hawthorne). Dessa maneira, cada turma de alunos foi designada a um grupo, o que caracteriza esse trabalho como um quase-experimento [CAMPBELL; STANLEY, 1996]. A turma 71, com 20 alunos $(\mathrm{M}=9, \mathrm{~F}=11)$, representou o grupo parcialmente gamificado; a turma 72, com 18 alunos $(\mathrm{M}=10, \mathrm{~F}=8)$, correspondeu ao grupo sem gamificação; e a turma 73, com 22 alunos $(\mathrm{M}=11, \mathrm{~F}=11)$, atuou como o grupo totalmente gamificado. É importante ressaltar que embora o design da avaliação fosse considerado um quaseexperimento, buscou-se controlar outras variáveis, por exemplo, o professor era o mesmo para as três turmas de matemática. Assim, o conteúdo, metodologia de ensino e a avaliação dos alunos eram os mesmos para todas as turmas. A Figura 2 apresenta o desenho experimental desta avaliação.

\section{Análise e Discussão dos Resultados}

A coleta de dados do experimento se deu através de logs do sistema e de um questionário elaborado pelos autores e aplicado ao final do experimento. A partir do log das interações dos alunos no sistema, foi possível identificar os estudantes mais propensos ao GTS e $\mathrm{HR}$, bem como a estimativa das intensidades desses comportamentos. O questionário teve como objetivo verificar o quanto que os alunos gostaram de utilizar o sistema e quais foram as suas opiniões sobre a gamificação e as características do tutor. 
VII Congresso Brasileiro de Informática na Educação (CBIE 2018)

Anais dos Workshops do VII Congresso Brasileiro de Informática na Educação (WCBIE 2018)

Todos os resultados foram testados com o teste de normalidade Shapiro-Wilk, apresentando distribuições significativamente não-normais. Assim, assumindo testes nãoparamétricos, os dados foram testados com o teste de Kruskal-Wallis.

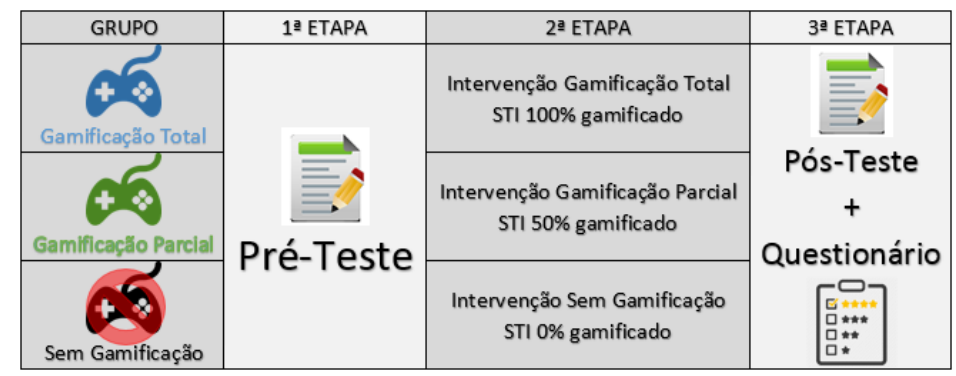

Figura 2. Desenho experimental da avaliação

\subsection{Gaming the System e Help Refusal}

Para identificar o nível desses comportamentos indesejados, foram elaboradas três fórmulas com o auxílio e validação de duas professoras de matemática que possuem de 10 a 20 anos de experiência em docência. Essas fórmulas estão disponíveis no Quadro 2.

Quadro 2. Fórmulas para identificar o Gaming the System e Help Refusal

\begin{tabular}{|c|c|c|}
\hline \multicolumn{2}{|c|}{ GAMING THE SYSTEM } & \multirow{2}{*}{ HELP REFUSAL } \\
\hline Tentativa e Erro & Help Abuse & \\
\hline $\mathrm{N}^{\circ}$ Erros seguidos & \multirow{3}{*}{$\frac{\mathrm{N}^{\mathrm{o}} \text { Dicas }+\mathrm{N}^{\mathrm{o}} \text { Erros }}{\mathrm{N}^{\mathrm{o}} \text { Erros } * 6}$} & \multirow{3}{*}{$\begin{array}{c}\mathrm{N}^{\mathrm{o}} \text { Erros seguidos } \\
\text { sem pedir dicas }\end{array}$} \\
\hline Média dos intervalos entre & & \\
\hline 10 & & \\
\hline
\end{tabular}

A primeira fórmula parte do princípio que os alunos podem errar até duas vezes seguidas um passo de uma equação sem caracterizar tentativa e erro, uma vez que o primeiro erro pode ter sido de digitação ou uma simples falta de atenção. A partir do terceiro erro seguido, há uma grande chance de caracterizar o comportamento de tentativa e erro; por esse motivo o numerador da fórmula é dividido por 3. Também é necessário considerar o tempo entre os erros seguidos, sendo que quanto mais próximos forem os erros seguidos, maior é a probabilidade desse comportamento (considerando a hipótese de que o aluno não analisou corretamente o passo errado antes da nova tentativa). Assim sendo, foi estimado que os alunos devem esperar pelo menos 10 segundos entre os erros seguidos para terem tempo suficiente de verificar o porquê de eles errarem. Finalmente, foi adicionado o número 1 na multiplicação para que os coeficientes de tentativa e erro não fiquem com valores muito altos caso o estudante tenha a média do tempo entre os erros inferior a 10 .

A segunda fórmula retorna a porcentagem de dicas utilizadas em relação a todos os erros cometidos pelos alunos. Quando um aluno erra um passo, ele também recebe uma dica, por esse motivo que o número de erros também é considerado no numerador da fórmula. Já o denominador segue o seguinte princípio: para cada um dos erros, o aluno poderia ter pedido quatro dicas, considerando que a maioria possui quatro níveis. No caso dos feedbacks de erro, eles geralmente possuem dois níveis, que somado com os outros quatro níveis das dicas temos seis possibilidades. Assim sendo, quanto maior a quantidade de dicas utilizadas, maior é a chance de Help Abuse. É importante ressaltar que é aceitável os alunos utilizarem até a metade das dicas disponíveis sem considerar abuso de ajuda. 
VII Congresso Brasileiro de Informática na Educação (CBIE 2018)

Anais dos Workshops do VII Congresso Brasileiro de Informática na Educação (WCBIE 2018)

Por último, a fórmula de Help Refusal baseia-se no seguinte princípio: quanto maior for o número de erros seguidos sem pedidos de dicas, maior será a chance de o aluno apresentar esse comportamento; se o aluno tivesse pedido ajuda provavelmente não teria errado tantas vezes seguidas.

A Tabela 1 mostra os resultados da aplicação dessas três fórmulas, indicando as médias, os resultados do teste de Krusal-Wallis e a comparação Posthoc entre os grupos.

Tabela 1. Testes Kruskall-Walis e Posthoc para os resultados das fórmulas

\begin{tabular}{|c|c|c|c|c|c|c|c|c|c|c|c|}
\hline \multirow{3}{*}{ Fórmula } & \multirow{2}{*}{\multicolumn{2}{|c|}{$\begin{array}{c}\text { Gamificação } \\
\text { Parcial }\end{array}$}} & \multirow{2}{*}{\multicolumn{2}{|c|}{$\begin{array}{c}\text { Gamificação } \\
\text { Total }\end{array}$}} & \multirow{2}{*}{\multicolumn{2}{|c|}{$\begin{array}{c}\text { Sem } \\
\text { Gamificação } \\
\end{array}$}} & \multirow{2}{*}{\multicolumn{2}{|c|}{ Kruskal-Wallis }} & \multicolumn{3}{|c|}{ Posthoc } \\
\hline & & & & & & & & & $\mathbf{T} \times \mathbf{S}$ & $\mathbf{T} \times \mathbf{P}$ & $\mathbf{P} \times \mathbf{S}$ \\
\hline & $\mathbf{M}$ & DP & $\mathbf{M}$ & DP & $\mathbf{M}$ & DP & H(2) & p & Dif. & Dif. & Dif. \\
\hline Tentativa e Erro & ,66 & 1,1703 & ,83 & 2,208 & 1,41 & 3,5751 & 10,26 &, 0059 & Sim & Não & Sim \\
\hline Help Refusal & 1,69 & 1,7034 & 1,69 & 2,62773 & 2,06 & 3,1174 &, 0014 & ,9993 & - & - & - \\
\hline
\end{tabular}

$\mathrm{M}=$ Média, DP = Desvio Padrão, H(2) = Estatísticas com 2 graus de liberdade, $\mathrm{p}=$ valor $\mathrm{p}$, Dif. = se a diferença é significativa Dados em negrito: significância estatística

Os resultados mostraram que os alunos da versão não gamificada apresentaram um maior índice do comportamento de Tentativa e Erro do que os grupos de gamificação total e parcial. Entretanto, não encontramos diferenças significativas entre os dois grupos gamificados para Tentativa e Erro. Acredita-se que os elementos adicionais não foram o suficiente para sobressair o grupo de gamificação parcial, devido à grande semelhança entre as duas versões. Em relação aos comportamentos de Help Abuse e Help Refusal, não conseguimos encontrar evidências que a gamificação pode impedir esses comportamentos.

\subsection{Questionário}

Nós elaboramos um questionário personalizado com perguntas que visam detectar o quanto os alunos gostaram de usar a respectiva versão do STI e os comportamentos específicos de Gaming the System e Help Refusal. Infelizmente, não foi possível utilizar um questionário já reconhecido na literatura, pois não temos conhecimento de instrumentos validados e gratuitos na língua portuguesa para identificar os comportamentos de ajuda e também engajamento em ambientes educacionais.

O questionário foi composto por 36 questões e asserções relacionadas à motivação dos alunos para o uso do tutor e suas opiniões sobre as dicas e gamificação, além de questões relacionadas aos comportamentos de HR e GTS. As questões objetivas seguiram uma escala Likert, com pontuações variando de 1 a 5 . Devido à limitação de espaço, optamos por mostrar apenas as questões mais relevantes na Tabela 2.

Conforme ilustrado na Tabela 2, os grupos sem gamificação e com gamificação total gostaram mais de utilizar a sua versão do sistema do que o grupo parcialmente gamificado. Para verificar se houve diferença significativa entre os grupos, o teste de Kruskal-Wallis foi aplicado a todos os dados da questão. Este teste foi escolhido em vez do ANOVA porque a aplicação do Shapiro-Wilk mostrou que os dados não seguem distribuição normal [FIELD et al., 2012].

As questões 2 e 3 estão diretamente relacionadas ao objetivo geral deste trabalho. O grupo não-gamificado apresentou a maior média, o que está de acordo com os resultados apresentados na seção anterior; no entanto, não há diferença estatisticamente significativa. No caso das questões 4 e 5, ambas estavam relacionadas apenas com as versões gamificadas do sistema. Mais uma vez, Shapiro-Wilk foi usado para testar a 
VII Congresso Brasileiro de Informática na Educação (CBIE 2018)

Anais dos Workshops do VII Congresso Brasileiro de Informática na Educação (WCBIE 2018)

normalidade. Os resultados do teste mostraram que os dados não seguem uma distribuição normal. Assim, o teste de classificação de Wilcoxon foi aplicado ao invés de usar um teste $\mathrm{t}$ [FIELD et al., 2012]. Os resultados do teste mostraram uma evidência marginalmente significativa para ambas as questões. Assim, há evidências que os alunos do grupo totalmente gamificado gostaram mais do sistema de pontuação do que o grupo com gamificação parcial.

Tabela 2. Principais perguntas do questionário e testes Kruskall-Walis, Posthoc e teste de comparação de Wilcoxon

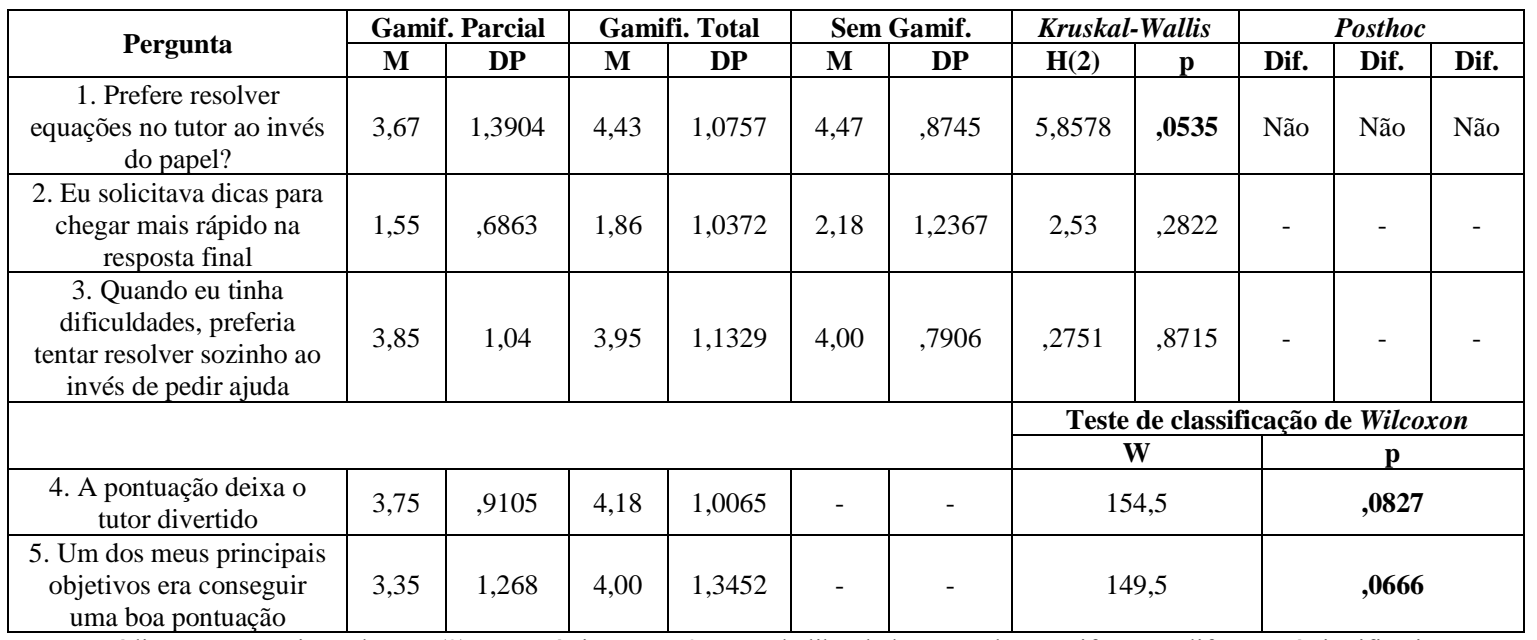

$\mathrm{M}=$ Média, DP = Desvio Padrão, H(2) = Estatísticas com 2 graus de liberdade, $\mathrm{p}=$ valor $\mathrm{p}$, Dif. = se a diferença é significativa. Dados em negrito: significância estatística

\subsection{Desempenho nos testes}

Tanto o pré-teste quanto o pós-teste foram compostos de 15 equações de mesmo peso. As notas foram calculadas na base 10 e os alunos poderiam ter acertos de $50 \%$ e $25 \%$. Um acerto de $50 \%$ indica que o estudante acertou o valor final, mas errou o sinal (positivo ou negativo). O segundo indica que o aluno acertou apenas a operação matemática principal da equação e errou o resultado final (o principal motivo desses erros foram em cálculos aritméticos básicos).

Utilizamos o teste de Shapiro-Wilk para testar a normalidade e o teste one-way ANCOVA para comparar as notas dos alunos nos pós-testes nas três condições, usando o desempenho no pré-teste como covariável. Não foi encontrada uma diferença com significância estatística $(\mathrm{F}(1,57)<0,01, \mathrm{p}=0,1807)$ entre os desempenhos no pós-teste para o grupo com gamificação total $(\mathrm{M}=5,78, \mathrm{DP}=1,9234)$, gamificação parcial $(\mathrm{M}=$ $6,39, \mathrm{DP}=2,6046)$ e sem gamificação $(\mathrm{M}=5,11, \mathrm{DP}=1,8615)$, indicando que não houve diferença entre os grupos. Entretanto, o gráfico da Figura 3 mostra que houve um ganho de aprendizado maior nos dois grupos gamificados ( $8 \%$ no grupo com gamificação total, $7 \%$ no grupo com gamificação parcial e menos de $1 \%$ no grupo sem gamificação). É possível que, embora haja um aparente ganho maior nos grupos gamificados, não conseguimos encontrar uma diferença estatística devido ao tamanho da amostra e/ou da curta duração do experimento.

\section{Conclusão}

Nós apresentamos uma proposta de solução para os comportamentos de Gaming the System e Help Refusal, que podem ser ocasionados a partir do uso inapropriado da 
VII Congresso Brasileiro de Informática na Educação (CBIE 2018)

Anais dos Workshops do VII Congresso Brasileiro de Informática na Educação (WCBIE 2018)

assistência inteligente oferecida pelos STIs. Este trabalho foi publicado como um artigo full paper na conferência EC-TEL 2018, com o título Exploring Gamication to Prevent Gaming the System and Help Refusal in Tutoring Systems.

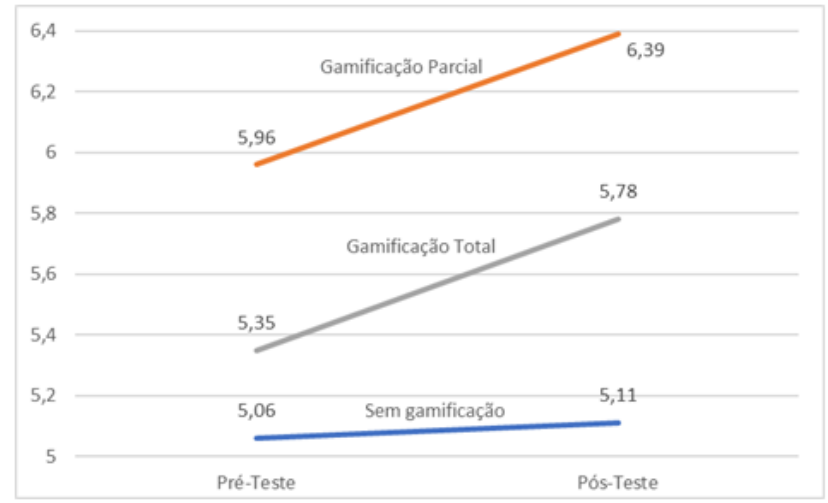

Figura 3. Desempenho dos estudantes nos pré-testes e pós-testes

O sistema desenvolvido tem como objetivo minimizar a ocorrência desses comportamentos a partir da Gamificação. Os elementos de Gamificação inseridos no tutor atuaram nos seguintes aspectos: disponibilização de dicas e erros gratuitos com base na complexidade do plano de aula e no número de equações; distribuição dos planos de aula em níveis de dificuldade e fases com nomes gamificados; e a otimização do sistema de pontuação já presente no sistema.

Para validar a proposta, foi realizada uma avaliação experimental envolvendo três turmas do $7^{\circ}$ ano de uma escola particular de São Leopoldo. Cada uma dessas turmas foi associada a um grupo, que podia ser totalmente gamificado, parcialmente gamificado e sem gamificação, constituindo assim um quase-experimento por não envolver uma distribuição 100\% aleatória [CAMPBELL; STANLEY, 1996].

Os resultados encontrados corroboram com o trabalho de [Pedro, 2016], mostrando que a gamificação pode ser utilizada para minimizar o Gaming the System. No entanto, [Pedro, 2016] comparou o tempo em que os alunos passaram lendo dicas e o enunciado do exercício, enquanto que neste trabalho comparamos a intensidade do comportamento em si. Nós também conseguimos identificar um impacto da gamificação no comportamento de tentativa e erro, porém não encontramos diferenças significativas entre as duas versões gamificadas. Isso possivelmente significa que os elementos adicionais não foram o suficiente para sobressair o grupo de gamificação parcial, devido à grande semelhança entre as duas versões. Além disso, nós não conseguimos mostrar que a gamificação pode reduzir os comportamentos de Help Abuse e Help Refusal. Entretanto, é possível elaborar explicações alternativas para os resultados encontrados: (i) as duas versões gamificadas são muito semelhantes; (ii) limitações do projeto de quaseexperimento (uma das turmas poderia ter alunos com mais facilidade em matemática); (iii) curta duração do experimento. Outra limitação deste estudo está relacionada com as fórmulas elaboradas: apesar de contarmos com o auxílio de professores de matemática que já usaram o tutor com os seus alunos, não validamos formalmente essas fórmulas. Embora essas fórmulas possam oferecer uma estimativa aproximada de Gaming the System e Help Refusal, futuros trabalhos poderiam desenvolver mecanismos validados para a detecção destes comportamentos. Como trabalho futuro, pretende-se estudar o impacto de diferentes elementos da gamificação nesses mesmos comportamentos, bem como no engajamento e aprendizado dos alunos. 
VII Congresso Brasileiro de Informática na Educação (CBIE 2018)

Anais dos Workshops do VII Congresso Brasileiro de Informática na Educação (WCBIE 2018)

\section{Referências}

Baker, R. S. Designing Intelligent Tutors That Adapt to When Students Game the System. Tese (Doutorado), Pittsburgh, PA, USA, 2006. AAI3241593

Campbell, D. T.; Stanley, J. C. Experimental and quasi-experimental designs for research. [S.1.]: R. McNally, 1966.

De Santana, S. J. et al. A quantitative analysis of the most relevant gamification elements in an online learning environment. In: Proceedings of the 25th International Conference Companion on World Wide Web. International World Wide Web Conferences Steering Committee, 2016. p. 911-916.

Faghihi, U. et al.. How Gamification Applies for Educational Purpose Specially with College Algebra. Procedia Computer Science, v. 41, p. 182-187, 2014.

Field, A., Miles, J., Field, Z.: Discovering statistics using R. Sage (2012)

González, C.; Mora, A.; Toledo, P. Gamification in intelligent tutoring systems. In: Proceedings of the Second International Conference on Technological Ecosystems for Enhancing Multiculturality. ACM, 2014. p. 221-225.

Huang, W. H. Y.; Soman, D. Gamification of education. Research Report Series: Behavioural Economics in Action, Rotman School of Management, University of Toronto, 2013.

Jaques, P. A., Seffrin, H., Rubi, G., de Morais, F., Ghilardi, C., Bittencourt, I. I., \& Isotani, S. (2013). Rule-based expert systems to support step-by-step guidance in algebraic problem solving: The case of the tutor PAT2Math. Expert Systems with Applications, 40(14), 5456-5465.

Kapp, K. M. The gamification of learning and instruction: game-based methods and strategies for training and education. John Wiley \& Sons, 2012.

Kulik, J. A., \& Fletcher, J. D. (2016). Effectiveness of Intelligent Tutoring Systems: A Meta-Analytic Review. Review of Educational Research, 86(1), 42-78.

Long, Y.; Aleven, V. Gamification of joint student/system control over problem selection in a linear equation tutor. In: International Conference on Intelligent Tutoring Systems. Springer International Publishing, 2014. p. 378-387.

Neto, A.; Da Silva, A. P.; Bittencourt, I. I. Uma análise do impacto da utilização de técnicas de gamificação como estratégia didática no aprendizado dos alunos. In: Brazilian Symposium on Computers in Education (Simpósio Brasileiro de Informática na Educação-SBIE). 2015. p. 667.

Pedro, L. Z. Uso de gamificação em ambientes virtuais de aprendizagem para reduzir o problema da externalização de comportamentos indesejáveis. 2016. Dissertação de Mestrado. Universidade de São Paulo.

Vanlehn, K. The Behavior of Tutoring Systems. International Journal of Artificial Intelligence in Education. V. 16, n. 3, p. 227-265, 2006. Amsterdam, The Netherlands, The Netherlands: IOS Press.

Woolf, B. P. Building intelligent interactive tutors: Student-centered strategies for revolutionizing e-learning. Morgan Kaufmann, 2010. 